\title{
The Role of a Teacher in Teaching Practice of Chinese Lacquerware He Yan
}

\author{
Sichuan Fine Arts Institute 108 Huang jue ping, Chongqing 400053, China \\ hhhyyyy1@163.com
}

Keywords: Chinese lacquer; Role; Teaching practice; Teacher

\begin{abstract}
The scholar has summarized the roles of a teacher while teaching in the existing literature. The teaching of lacquerware creation at Sichuan Fine Arts Institute is quite different from that of science and technology. The specific requirements to a teacher in lacquerware discipline based on the author's working experience are presented in this paper.
\end{abstract}

\section{Introduction}

Teaching is a more complex profession than most people realize and teacher plays an important role in students' knowledge gaining. Teachers give students the one-on-one help they need, right when they need it. Teachers can do almost anything within a course, including adding or changing the activities and grading students. The roles of teacher have been divided as 9 kinds of characters in literature 1 [1]. It is very difficult to fill every role and to do everyone well. However, effective teachers embrace each of these roles and work continuously to improve the areas in which they are weakest.

Art is a diverse range of human activities in creating visual, auditory or performing artworks, expressing the author's imaginative or technical skill, intended to be appreciated for their beauty or emotional power $[2,3]$. In their most general form these activities include the production of works of art, the criticism of art, the study of the history of art, and the aesthetic dissemination of art. The Chinese lacquerware is a kind of art production and it has thousands of year's history in China [4-8]. The Sichuan Fine Arts Institute has launched discipline of Chinese Lacquerware. The role of teacher in art is somewhat different with teacher in science and technology. The role of teacher in Chinese lacquerware at Sichuan Fine Arts Institute is discussed in this paper based on author' teaching practice and literature 1 [1].

\section{Roles of a Teacher in Lacquerware}

Teacher as Classroom Manager. A teacher must be an effective classroom manager. This area includes organization, preparation, and student discipline. True classroom management is proactive not reactive meaning that the teacher has the foresight to head a problem off before it becomes something bigger. The teaching activity on Chinese lacquerware is different with that of science and technology. The dominant activity is creation of an art work under the instruction of teacher and art production of each student is quite different from each other. Apparently, it looks out of order in the classroom (see Fig. 1) the teacher must manages the classroom as a manger. 


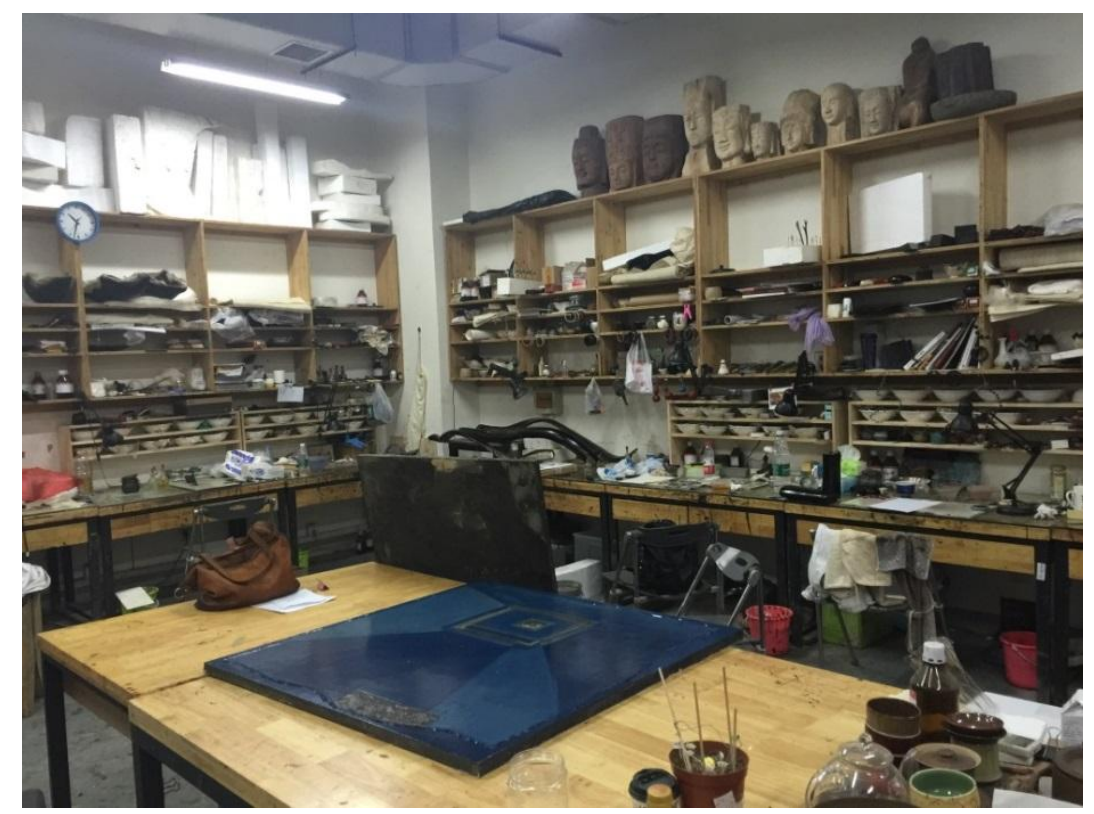

Figure 1. The classroom of Lacquerware Creation

Teacher as Collaborator. Collaboration is two or more people working together to solve a problem or improve a targeted area. Communication is simply exchanging information or concerns with another person. The creation of Chinese lacquerware is very complicated, there are 26 steps to finish a lacquerware [9]. The teacher must guide the students to finish the modelling, bodiless and other steps. The teacher's idea is melted with that of students, so the teacher must continuously communicate with their students, and collaborates with students on a daily basis. Please see the collaboration of teacher and students in Fig. 2.

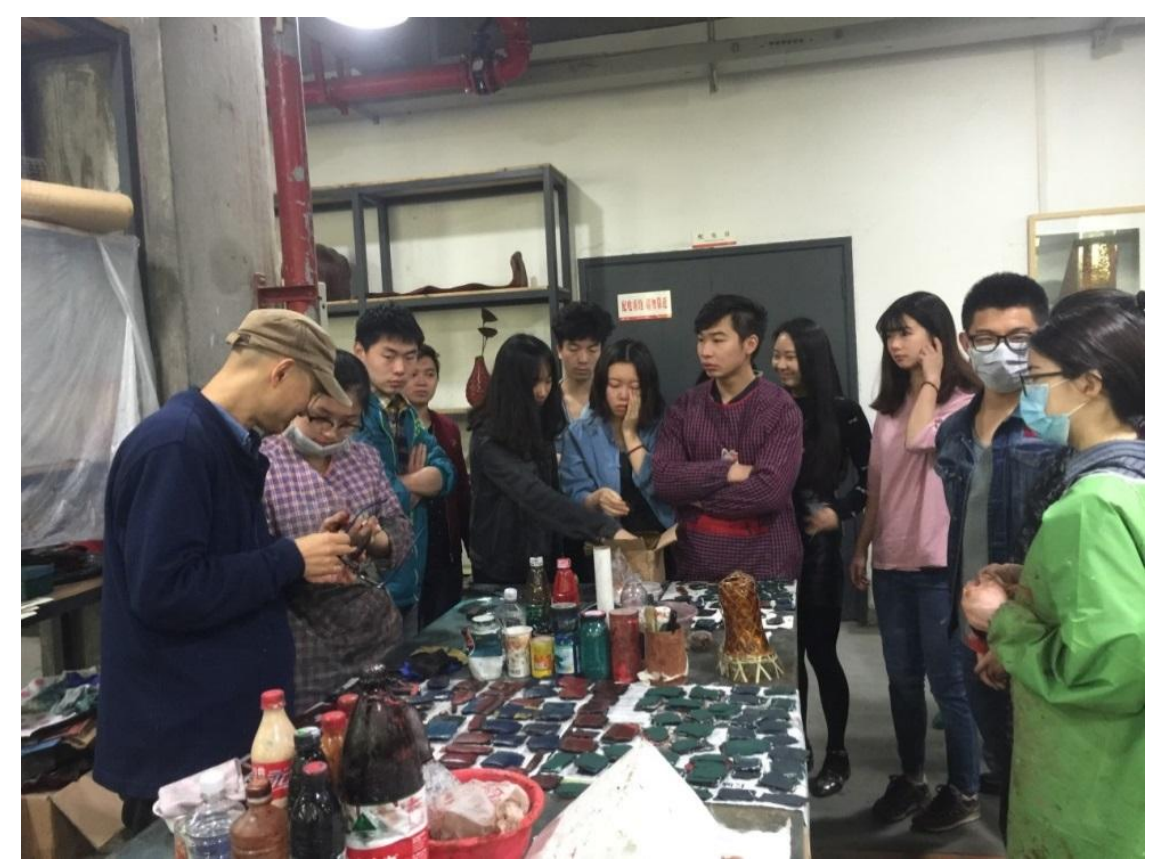

Figure 2. The collaboration of teacher with students

Teacher as Content Expert. Teachers must become experts on the grade level and content which they teach. A teacher must be able to convince their students effectively that they genuinely know what they are talking about, or their students will not buy in. Teachers become experts through studying and preparation. The contemporary lacquerware is not only the inheriting of traditional craft, but also an innovation. The traditional lacquerware includes boxes, tableware, 
buttons and even coffins painted with lacquer. But modern style lacquerware is not restricted to Chinese traditional culture; the contemporary abstract art should also be applied to the lacquerware creation (see Fig. 3). The teacher must have a good education background on Chinese traditional culture, contemporary art, and modern art. The teacher has to keep on reading, thinking, creating and attending exhibition to make sure that he/she is an expert in his/her teaching area.

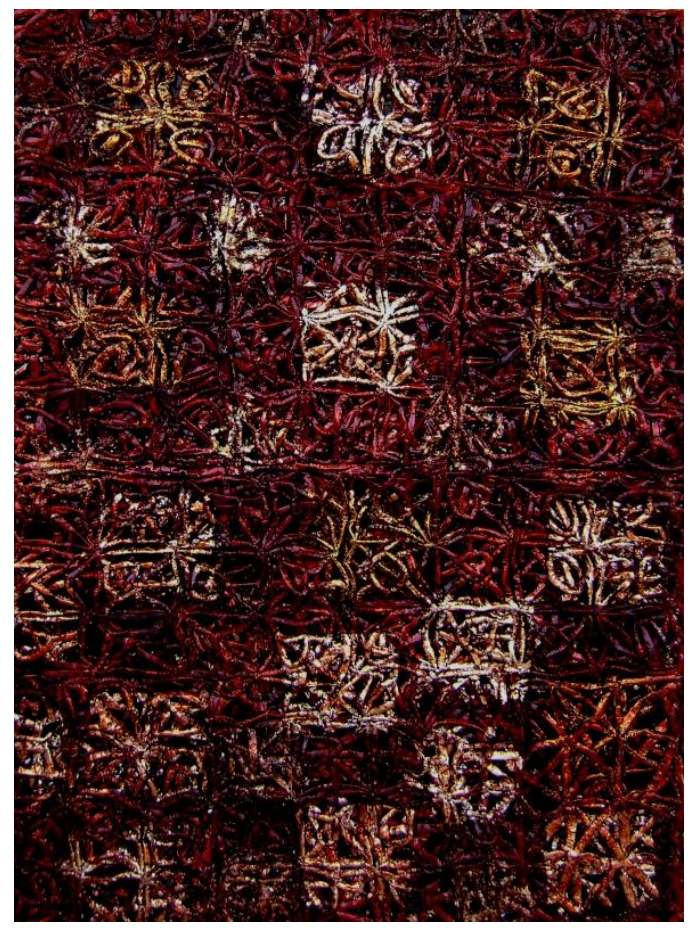

Figure 3. Lacquerware in the style of contemporary abstract art

Teacher as Facilitator. The art work always has been produced accidentally, because of the interactive and influence between the artist and the creative object. There is not an invariant way to guide the different students. The teacher must have many different methods and utilizing various instructional strategies. One thing that makes teaching such an interesting and diverse field is that there is more than one way to do this job effectively. What works well for one group of students, may not necessarily work for another. Teachers must be flexible in their approach and willing to adjust to those with which they are working. The final production of different students exhibits quite different effects (See Fig. 4) 

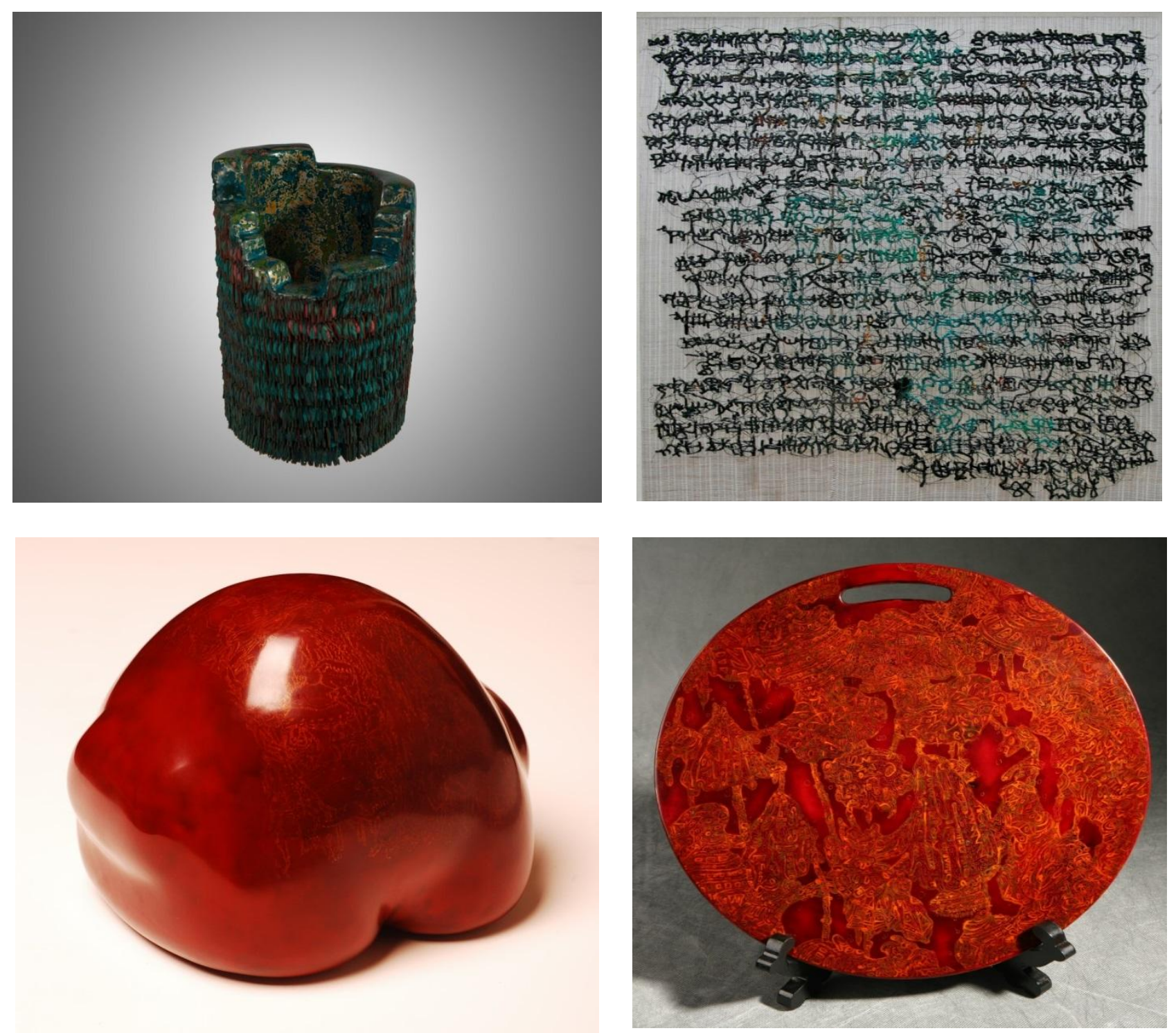

Figure 4. The art productions created by different students

Teacher as Mentor. Teachers spend a considerable amount of time working with their students. Because of this it is natural for relationships and bonds to form with certain students. Every student is different. Some students simply require more attention than others. Those are the students with which we typically spend more time. Eventually, the trust is formed and sometimes the teacher can step into the role of mentor. Many students who have never been successful academically, finally attained success because a teacher saw something in them and took the time to take on a mentoring role that led to that breakthrough.

Teacher as Protector. The Chinese lacquer, cashew and polyurethane lacquer are three main kinds of materials applied in the current lacquerware technique. The deficiencies of these materials are obvious. Firstly and most importantly, these materials are harmful to the human's health. Traditionally, Chinese lacquer, cashew and polyurethane are deemed as non-toxic and no harm for the body, but actually this point of view is completely wrong [10]. For example, the Chinese lacquer can cause skin allergy and ulcer; even severely affected patience should be hospitalized and treated. The principle component of cashew is cashew oil, but without additives containing Benzenne, the effects of cashew is greatly decreased. The Benzenne is harmful to the human's health. The polyurethane can damage human's respiratory passageways and livers. A teacher's greatest role is to ensure the safety and security of every student. At school, teachers must ensure that they provide their students with a safe learning environment. They must monitor students at all times throughout the school day and be prepared to remove them from a threatening situation. 


\section{Conclusion}

The scholar has summarized the roles of a teacher while teaching in the existing literature. The teaching of lacquerware creation at Sichuan Fine Arts Institute is quite different from that of science and technology. The specific requirements to a teacher in lacquerware discipline based on the author's working experience are presented in this paper.

\section{References}

[1] http://teaching.about.com/od/pd/fl/Role-of-the-Teacher.htm

[2] Information on http://www.weld.labs.gov.cn

[3] Information on http://www.merriam-webster.com/dictionary/art

[4] L, Jiang. Usage of the lifetime of Lacquer Culture. Decoration. 2(2012)14-16

[5] W. Zheng, J. Liu, et al., Preparation of Green Tung Paint. Hubei Agricultural Sciences. 50(2011)2724-2726

[6] R. Bagley, Shang Archaeology. The Cambridge History of Ancient China: From the Origins of Civilization to 221 B.C., Cambridge University Press, 1999, pp. 178-178.

[7] L. Li, China's Cultural Relics, Cambridge: Cambridge University Press, 2011, pp. 139-140.

[8] F. Zhang, The origin of Chinese Lacquer Crafts. Journal of Chinese Lacquer. 5(2008)21-37

[9] Y. He, X. Zhu, The craft of Chinese lacquerware. Chongqing University Press, 2013, pp. 1-49.

[10]B. Li, Technique and Art of Lacquer Painting: Research on Cultural Values and Promotion of Chu Lacquer Art in Wuling Mountains Area. Design Research. 4(2014) 50-53 